\title{
Performance analysis of dual-hop satellite relay networks with hardware impairments and co-channel interference
}

\author{
Guo Kefeng* (D), Guo Daoxing, Huang Yuzhen and Zhang Bangning
}

\begin{abstract}
In this paper, we investigate the impact of hardware impairments on the outage performance and the instantaneous capacity of dual-hop decode-and-forward (DF) satellite relay networks in the presence of co-channel interference. Specifically, the exact closed-form expression for the outage probability and the analytical expression for the instantaneous capacity of the system are derived over a shadowed-Rician fading channel, which provides a fast means to evaluate the impact of hardware impairments on the system performance. Our results demonstrate that the outage floor occurs and the capacity upper bound appears when the hardware impairments exist. In addition, simulation results were provided to verify the correctness of the analytical results.
\end{abstract}

Keywords: Satellite relay network, Hardware impairments, Co-channel interference, Outage probability, Instantaneous capacity

\section{Introduction}

A relay is used to improve the coverage, reliability, and quality of service in wireless systems, which has become a hot research topic over the past decade [1-4]. A satellite is used as the relay to help a source transmit a signal in a farther distance when the source itself cannot transmit directly. Satellite relay communication is capable of providing seamless connectivity and high-speed broadband access for worldwide users, especially in the fields of broadcasting, disaster relief, and navigation, where the deployment of wired and wireless terrestrial networks is not economically applicable.

The links between the ground and a satellite are usually modeled by composite fading distribution to describe more accurately the amplitude fluctuation (large scale) of the transmitted signal's envelope. To this end, the shadowed-Rician distribution has been proposed in [5]. It approaches the line-of-sight (LOS) communication using the Rician distribution, while the fluctuation of the signal's amplitude is Nakagami-m distributed. Hence, it agrees

*Correspondence: guokefeng.cool@163.com

College of Communications Engineering, PLA University of Science and

Technology, Guanghua Road, Nanjing, China very well with experimental data, whereas it demonstrates quite a manageable mathematical tractability.

Capitalizing on the latter features of the shadowedRician distribution, an analytical performance evaluation of dual-hop amplify-and-forward (AF) systems with a satellite relay, was provided in [6] and [7]. In [8] and [9], a single-antenna land mobile satellite (LMS) system was investigated, where the source is a satellite and the relay/destination are terrestrial nodes. Furthermore, a similar LMS system with the source-to-relay satellite link was investigated in [10].

However, most research in the area of satellite relaying assumes that the transceiver hardware of the network node is perfect. This cannot be achieved in practical communication systems. In practice, hardware suffers from several types of impairments, e.g., phase noise, I/Q imbalance, and high power amplifier (HPA) nonlinearities [11-13]. The impact of hardware impairments on singlehop wireless communication was broadly investigated in [14-16]. In addition, as indicated in [17-20], there is a fundamental capacity ceiling due to the hardware impairments, which cannot be improved by increasing the transmission power. In [17], the authors analyzed the 
performance of multiple-input-multiple-output (MIMO) system; the capacity and the outage probability of the system were derived. In [18], the authors analyzed the system performance of the full-duplex relaying network and found that the system capacity had an upper bound in the presence of hardware impairments. In [19], the opportunistic and partial relaying selection was used in which a new way was derived to obtain the closed-form expression for the system performance. In [20], the authors analyzed the impact of hardware impairments on the fixedgain AF relaying Nakagami-m fading channels. Above all, the system channel they analyzed was just a Rayleigh or Nakagami-m channel; there are scarcely any papers that analyzed the effect of hardware impairments on the shadowed-Rician channel.

Although the aforementioned works have significantly improved our understanding on the performance and benefits of a satellite relay network, they only considered a noise-limited scenario and the ideal case of no co-channel interference (CCI) at the destination, which are unrealistic due to the current aggressive reuse of spectrum resources. In recent years, a great deal of research has focused on the performance of conventional terrestrial systems in the presence of CCI over Rayleigh or Nakagami-m fading channels [21-23]. Due to the reuse of spectrum resources in practice, the major challenge facing satelliteterrestrial networks is the problem of interference from inter-component and/or intra component networks [24]. The authors in [25] first investigated the performance of satellite-terrestrial networks with CCI at the destination for the single antenna scenario and analyzed the system performance.

As described above, we find that it is significant to analyze the impact of hardware impairments and CCI on the satellite relay communication network in shadowedRician channel. Until now, to the best of the authors' knowledge, there is scarcely any paper that analyzes the impact of hardware impairments on a satellite relay network with CCI. To fill this gap, we herein investigate the joint impact of hardware impairments and CCI on the performance of a dual-hop satellite relay network.

Our main contributions are summarized as follows:

- Firstly, the closed-form expression for the outage probability is derived which is applicable to cases involving an arbitrary number of interferers, antennas, and hardware impairments, and hence, the derived expression provides a fast means to investigate the system performance.

- Secondly, the analytical expression for the instantaneous capacity of the system, which can be used to efficiently evaluate the instantaneous capacity at high signal-to-noise-rates (SNRs), is derived.
- Thirdly, in this paper, the system performance is analyzed under two channel conditions, that is, channel parameters are integers or arbitrary values.

The rest of this paper is organized as follows. In Section 2, the model we analyzed is briefly introduced. In Section 3, the cumulative distribution function (CDF) of the end-to-end signal-to-interference-plus-noise-anddistortion-ratio (SINDR), the exact expression for the outage probability, and the analytical expression for the instantaneous capacity are derived. In addition, some system performance metrics are provided. In Section 5, the analytical results are presented. Finally, in Section 5, a brief summary of our work for this paper is presented. In the Appendix, the detailed proofs for some Lemmas and Theorems are given.

\section{System and channel models}

As shown in Fig. 1, we consider a satellite relay network, where the source $(S)$ communicates with destination $(D)$ with the help of the satellite relay $(R) . S$ is equipped with $N_{1}$ antennas, while $D$ and $R$ are equipped with a single antenna, which is a scenario applicable to satellite relay networks. We assume that the channels of the first hop and second hop follow the shadowed-Rician fading. In addition, we assume that the signal received at D is corrupted by co-channel interference. To achieve a good trade-off between the performance and implementation cost, the transmit-antenna-selection (TAS) is used at the transmitter.

The communication between $S$ and $D$ takes place in two time slots. In the first time slot, $S$ sends its signal to $R$. Similar to [2], when the hardware of each node is ideal, the received signal at $R$ is given by $y_{1 i}=h_{1 i} s+v_{r}$, where $y_{1 i}$ is the received signal transmitted by the $i$-th antenna of $S, h_{1 i}$ is the channel coefficient between the $i$-th antenna of $S$ and $R$, which follows the shadowed-Rician random variable (RV). $s_{1 i}$ is the signal from the $i$-th antenna of $S$ with average power $P_{1 i}=E\left[\left|s_{1 i}\right|^{2}\right] \cdot v_{r}$ is the additive white Gaussian noise (AWGN) which is represented as $v_{r} \sim$ $\mathcal{C N}\left(0, \delta_{r}^{2}\right)$ at $R$. However, when the node of the system has hardware impairments, the received signal changes, which is given by

$$
y_{1 i}=h_{1 i}\left(s_{1 i}+\eta_{1 i}\right)+v_{r}\left(i=1, \ldots, N_{1}\right)
$$

where $\eta_{1 i}$ denotes the distortion noise with zero mean and variance $k_{1 i}^{2} P_{1 i}$ and $k_{1 i}$ is sufficient to characterize the aggregate level of impairments of the $i$-th antenna of $S \rightarrow$ $R$ channel [26].

In the second time slot, the signal is forwarded to $D$ by $R$. The decode-and-forward (DF) relaying protocol is 


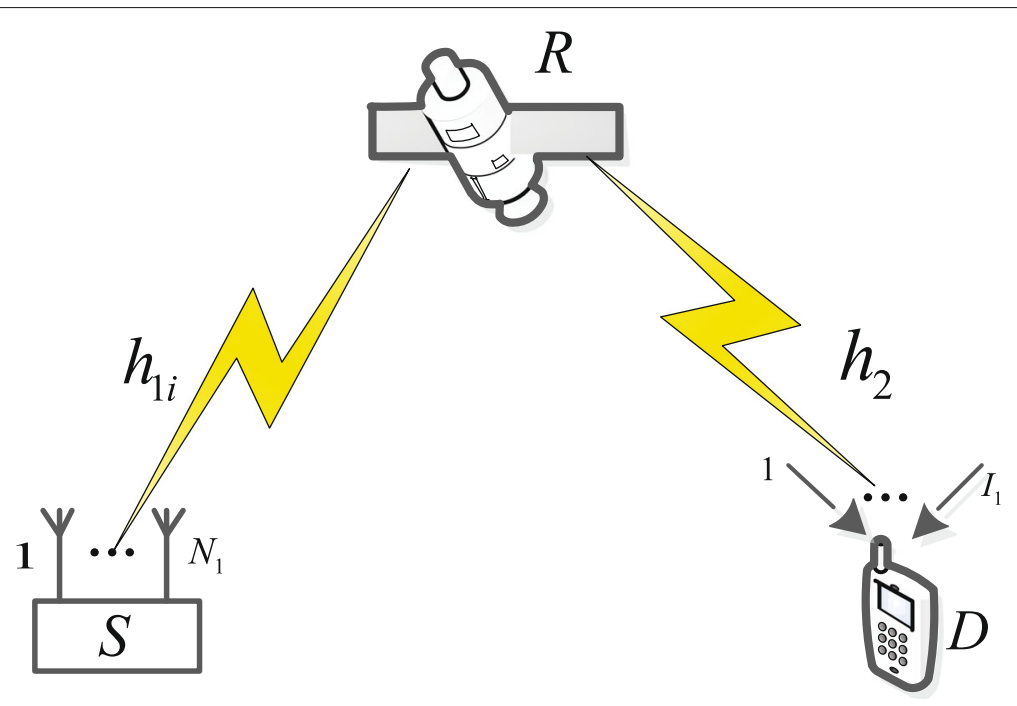

Fig. 1 The system model

always used in the satellite relay communication. Hence, the signal received at $D$ is given by

$$
y_{2}=h_{2}\left(y_{r}+\eta_{2}\right)+\sum_{j=1}^{I_{1}} g_{j}\left(s_{j}+\eta_{j}\right)+v_{d}
$$

where $h_{2}$ is the independent but non-identically distributed shadowed-Rician RV between $R$ and $D$ and $g_{j}$ denotes the channel coefficient between the $j$-th interference $(I)$ and $D$ which is an independent but non-identically distributed Rayleigh RV. $y_{r}$ is the signal received from $R$ with average power $P_{2}=E\left[\left|y_{r}\right|^{2}\right], s_{j}$ is the signal from the $j$-th interference with power $P_{j}=E\left[\left|s_{j}\right|^{2}\right]$, $v_{d}$ is the AWGN which is represented as $v_{d} \sim \mathcal{C N}\left(0, \delta_{d}^{2}\right)$ at $D, \eta_{2}$ denotes the distortion noise with zero mean and variance $k_{2}^{2} P_{2}, \eta_{j}$ denotes the distortion noise with zero mean and variance $k_{j}^{2} P_{j}$ of the $I$, and $k_{2}$ and $k_{j}$ are sufficient to characterize the aggregate level of impairments of the $R \rightarrow D$ channel and $I \rightarrow D$ channel, respectively [26]. To simplify the analysis, we assume that the hardware impairment level of the $I \rightarrow D$ channel has the same value as $k_{1}=\ldots=k_{j}=\ldots=k_{I_{1}}, j=1, \ldots, I_{1}$.

\section{System performance}

In this section, we present a comprehensive performance investigation of the system described in the previous section. Specifically, the description of the shadowedRician channel, the closed-form expression of the outage performance, and the analytical expression of the instantaneous capacity of the considered system with hardware impairments are derived.

\subsection{The end-to-end SINDR of the system}

From (1), the SNDR of $y_{1 i}$ can be expressed as

$$
\begin{aligned}
\gamma_{1 i} & =\frac{\left|h_{1 i}\right|^{2} P_{1 i}}{\left|h_{1 i}\right|^{2} k_{1 i}^{2} P_{1 i}+\delta_{r}^{2}} \\
& =\frac{\lambda_{1 i}}{\lambda_{1 i}\left(1+\Delta_{1 i}\right)+1}
\end{aligned}
$$

where $\lambda_{1 i}=\frac{\left|h_{1 i}\right|^{2} P_{1 i}}{\delta_{r}^{2}}$ and $k_{1 i}^{2}=\Delta_{1 i}$.

Because the TAS protocol is considered, the SNDR of the first hop is given by

$$
\gamma_{1}=\max _{i=1, \ldots, N_{1}}\left(\gamma_{1 i}\right)
$$

In the same way, from (2), the SINDR of the second hop is given by

$$
\begin{aligned}
\gamma_{2} & =\frac{\left|h_{2}\right|^{2} P_{2}}{\left|h_{2}\right|^{2} k_{2}^{2} P_{2}+\sum_{j=1}^{I_{1}}\left|g_{j}\right|^{2} P_{j}\left(1+k_{j}^{2}\right)+\delta_{d}^{2}} \\
& =\frac{\lambda_{2}}{\lambda_{2} \Delta_{2}+\sum_{j=1}^{I_{1}} \lambda_{j}\left(1+\Delta_{j}\right)+1}
\end{aligned}
$$

where $\lambda_{2}=\frac{\left|h_{2}\right|^{2} P_{2}}{\delta_{d}^{2}}, \lambda_{j}=\frac{\left|g_{j}\right|^{2} P_{j}}{\delta_{d}^{2}}, k_{2}^{2}=\Delta_{2}$ and $k_{j}^{2}=\Delta_{j}$.

According to the DF relaying protocol, the end-to-end SINDR of the system is given by

$$
\gamma_{e}=\min \left(\gamma_{1}, \gamma_{2}\right)
$$




\subsection{The shadowed-Rician channel}

Because the $S \rightarrow R$ and $R \rightarrow D$ channels are modeled as the shadowed-Rician fading distribution [5], the probability density function (PDF) of $\lambda_{1 i}$ or $\lambda_{2}$ is given by

$$
f_{\lambda_{l p}}(x)=\frac{\alpha_{l p}}{\bar{\lambda}_{l p}} e^{-\frac{\beta_{l p}}{\bar{\lambda}_{l p}} x}{ }_{1} F_{1}\left(m_{l p} ; 1 ; \frac{\delta_{l p}}{\bar{\lambda}_{l p}} x\right), x>0
$$

where $\{l, p\} \in\{\{1, i\},\{2\}\}, \bar{\lambda}_{l p}$ is the average SNR of each link, $\alpha_{l p} \triangleq \frac{\left(\frac{2 b_{l p} m_{l p}}{2 b_{l p} m_{l p}+\Omega_{l p}}\right)^{m_{l p}}}{2 b_{l p}}, \quad \beta_{l p} \triangleq \frac{1}{2 b_{l p}}, \quad \delta_{l p} \triangleq \frac{\Omega_{l p}}{2 b_{l p}\left(2 b_{l p} m_{l p}+\Omega_{l p}\right)}$, $\Omega_{l p}, 2 b_{l p}$ and $m_{l p} \geq 0$ correspond to the average power of the LOS component, the average power of the multipath component, and the fading severity parameter ranging from 0 to $\infty$, respectively. In the following, we consider the two cases of $m_{l p}$ being either an integer value or an arbitrary value. In $(7),{ }_{1} F_{1}\left(a_{1} ; b_{1} ; z\right)$ denotes the confluent hypergeometric function defined in [27].

\subsubsection{Integer $m_{l p}$ value case}

If $m_{l p}$ is an integer, with the aid of [28], ${ }_{1} F_{1}\left(m_{l p} ; 1 ; \frac{\delta_{l p}}{\bar{\lambda}_{l p}} x\right)$ can be represented as (8)

${ }_{1} F_{1}\left(m_{l p} ; 1 ; \frac{\delta_{l p}}{\bar{\lambda}_{l p}} x\right)=e^{\frac{\delta_{l p}}{\bar{\lambda}_{l p}} x} \times \sum_{k_{l p}=0}^{m_{l p}} \frac{\left(-\delta_{l p}\right)^{k_{l p}}\left(1-m_{l p}\right)_{k_{l p}} x^{k_{l p}}}{\left(\bar{\lambda}_{l p}\right)^{k_{l p}}\left(k_{l p} !\right)^{2}}$

where $(\cdot)_{q}$ is the Pochhammer symbol.

Substituting (8) into (7), we have

$$
\begin{aligned}
f_{\lambda_{l p}}(x)= & \alpha_{l p} \sum_{k_{l p}=0}^{m_{l p}-1} \frac{\left(1-m_{l p}\right)_{k_{l p}}\left(-\delta_{l p} x\right)^{k_{l p}}}{\left(k_{l p} !\right)^{2}\left(\bar{\lambda}_{l p}\right)^{k_{l p}+1}} \\
& \exp \left(-\left(\frac{\beta_{l p}-\delta_{l p}}{\bar{\lambda}_{l p}}\right) x\right)
\end{aligned}
$$

Hence, the CDF of $\lambda_{l p}$ can be given by

$$
\begin{aligned}
F_{\lambda_{l p}}\left(x_{0}\right)= & \alpha_{l p} \sum_{k_{l p}=0}^{m_{l p}-1} \frac{\left(-\delta_{l p}\right)^{k_{l p}}\left(1-m_{l p}\right)_{k_{l p}}}{\left(k_{l p} !\right)^{2}\left(\beta_{l p}-\delta_{l p}\right)^{k_{l p}+1}} \\
& \gamma\left(k_{l p}+1,\left(\frac{\beta_{l p}-\delta_{l p}}{\bar{\lambda}_{l p}}\right) x_{0}\right)
\end{aligned}
$$

\subsubsection{Arbitrary $m_{l p}$ value case}

If $m_{l p}$ is an arbitrary value, with the aid of [27], ${ }_{1} F_{1}\left(m_{l p} ; 1 ; \frac{\delta_{l p}}{\bar{\lambda}_{l p}} x\right)$ can be rewritten as

$$
{ }_{1} F_{1}\left(m_{l p} ; 1 ; \frac{\delta_{l p}}{\bar{\lambda}_{l p}} x\right)=\sum_{k_{l p}=0}^{\infty} \frac{\left(m_{l p}\right)_{k_{l p}}\left(\delta_{l p} x\right)^{k_{l p}}}{\left(\bar{\lambda}_{l p}\right)^{k_{l p}}\left(k_{l p} !\right)^{2}}
$$

Now, to this end, by substituting (11) into (7), we have

$$
f_{\lambda_{l p}}(x)=\alpha_{l p} e^{-\frac{\beta_{l p}}{\bar{\lambda}_{l p}} x} \sum_{k=0}^{\infty} \frac{\left(m_{l p}\right)_{k_{l p}}\left(\delta_{l p} x\right)^{k_{l p}}}{\left(\bar{\lambda}_{l p}\right)^{k_{l p}+1}\left(k_{l p} !\right)^{2}}
$$

Hence, the CDF of $\lambda_{l p}$ is given by

$$
F_{\lambda_{l p}}\left(x_{0}\right)=\alpha_{l p} \sum_{k_{l p}=0}^{\infty} \frac{\left(m_{l p}\right)_{k_{l p}}}{\left(k_{l p} !\right)^{2}} \frac{\delta_{l p}^{k_{l p}}}{\beta_{l p}^{k_{l p}+1}} \gamma\left(k_{l p}+1, \frac{\beta_{l p}}{\bar{\lambda}_{l p}} x_{0}\right)
$$

\subsection{The outage performance}

According to [1], the outage probability is defined as the probability that the instantaneous end-to-end SINDR falls below a predefined threshold $x_{0}$. With the help of (6), the outage probability of the system has the following expression.

$$
\begin{aligned}
P_{\text {out }}\left(x_{0}\right) & =\operatorname{Pr}\left(\gamma_{e} \leq x_{0}\right) \\
& =\operatorname{Pr}\left[\min \left(\gamma_{1}, \gamma_{2}\right) \leq x_{0}\right]
\end{aligned}
$$

where $P_{\text {out }}\left(x_{0}\right)$ is the outage probability of the system. With the aid of [27], $P_{\text {out }}\left(x_{0}\right)$ can be represented as

$$
P_{\text {out }}\left(x_{0}\right)=F_{\gamma_{1}}\left(x_{0}\right)+F_{\gamma_{2}}\left(x_{0}\right)-F_{\gamma_{1}}\left(x_{0}\right) F_{\gamma_{2}}\left(x_{0}\right)
$$

Next, $F_{\gamma_{1}}\left(x_{0}\right)$ and $F_{\gamma_{2}}\left(x_{0}\right)$ are derived respectively.

Lemma 1 The CDF of $\gamma_{1}$ is derived as (16) and (17) respectively.

Proof With the help of (4), (10), and (13), $F_{\gamma_{1}}\left(x_{0}\right)$ can be represented as (16), and (17) in both cases, which are shown at the top of this page and next page, respectively. 


$$
\begin{aligned}
& F_{\gamma_{1}}\left(x_{0}\right)= \\
& \begin{cases}{\left[\alpha_{1 i} \sum_{k_{1 i}=0}^{m_{1 i}-1} \frac{\left(-\delta_{1 i}\right)^{k_{1 i}}\left(1-m_{11}\right)_{1 i}}{\left(\beta_{1 i}-\delta_{1 i}\right)^{k_{1 i}+1}\left(k_{1 i} !\right)^{2}} \gamma\left(k_{1 i}+1,\left(\frac{\beta_{1 i}-\delta_{1 i}}{\bar{\lambda}_{1 i}}\right) \frac{x_{0}}{1-\Delta_{1 i} x_{0}}\right)\right]^{N_{1}}} & , x_{0}<\frac{1}{\Delta_{1 i}} \\
1, & x_{0} \geq \frac{1}{\Delta_{1 i}}\end{cases} \\
& F_{\gamma_{1}}\left(x_{0}\right)= \begin{cases}{\left[\alpha_{1 i} \sum_{k_{1 i}=0}^{\infty} \frac{\left(m_{1 i}\right)_{k_{1}}}{\left(k_{1 i} !\right)^{2}} \frac{\delta_{1 i}^{k_{1 i}}}{\beta_{1 i} k_{i}+1} \gamma\left(k_{1 i}+1, \frac{\beta_{1 i}}{\bar{\lambda}_{1 i}} \frac{x_{0}}{1-\Delta_{1 i} x_{0}}\right)\right]^{N_{1}}} & , x_{0}<\frac{1}{\Delta_{1 i}} \\
1, & x_{0} \geq \frac{1}{\Delta_{1 i}}\end{cases}
\end{aligned}
$$

Lemma 2 With the help of (5), $F_{\gamma_{2}}\left(x_{0}\right)$ is given by (19) and (20), respectively.

Proof $F_{\gamma_{2}}\left(x_{0}\right)$ can be presented as

$$
\begin{aligned}
F_{\gamma_{2}}\left(x_{0}\right) & =F\left(\frac{\lambda_{2}}{\lambda_{2} \Delta_{2}+\sum_{j=1}^{I_{1}} \lambda_{j}\left(1+\Delta_{j}\right)+1} \leq x_{0}\right) \\
& =F\left(\lambda_{2} \leq \frac{\sum_{j=1}^{I_{1}} \lambda_{j}\left(1+\Delta_{j}\right) x_{0}+x_{0}}{1-\Delta_{2} x_{0}}\right) .
\end{aligned}
$$

With the help of (10), (13), and (18), the closed-form expression for $F_{\gamma_{2}}\left(x_{0}\right)$ is derived as (19) and (20) respectively; a detailed proof is given in the Appendix.

$$
\begin{aligned}
& F_{\gamma_{2}}\left(x_{0}\right)= \\
& \begin{cases}\sum_{k_{2}=0}^{m_{2}-1} \sum_{i=1}^{\rho(A)} \sum_{j=1}^{\tau_{i}(A)} \frac{\alpha_{2}\left(-\delta_{2}\right)^{k_{2}}\left(1-m_{2}\right)_{k_{2}}}{\Gamma\left(k_{2}+1\right)\left(\beta_{2}-\delta_{2}\right)^{k_{2}+1}} \chi_{i, j}(A) \frac{\mu_{\langle i\rangle}^{-j}}{(j-1) !}\left\{\Gamma(j) \mu_{\langle i\rangle}^{j}-e^{-\frac{\left(\beta_{2}-\delta_{2}\right)\left(1+\Delta_{j}\right) x_{0}}{\left(1-\Delta_{2} x_{0}\right) \bar{\lambda}_{2}}}\right. & \\
\left.\sum_{m=0}^{k_{2}} \sum_{v=0}^{m}\left(\begin{array}{l}
m \\
v
\end{array}\right) \frac{\left(1+\Delta_{j}\right)^{v} \Gamma(v+j)}{m !}\left[\frac{\left(\beta_{2}-\delta_{2}\right) x_{0}}{\left(1-\Delta_{2} x_{0}\right) \bar{\lambda}_{2}}\right]^{m}\left[\frac{\left(\beta_{2}-\delta_{2}\right) x_{0}}{\left(1-\Delta_{2} x_{0}\right) \bar{\lambda}_{2}}+\frac{1}{\mu_{(i)}}\right]^{-(v+j)}\right\} & , x_{0}<\frac{1}{\Delta_{2}} \\
1, & x_{0} \geq \frac{1}{\Delta_{2}}\end{cases} \\
& F_{\gamma_{2}}\left(x_{0}\right)=
\end{aligned}
$$



Theorem 1 When $m_{l p}$ is an integer, $F_{\gamma_{e}}\left(x_{0}\right)$ can be given by (21). 
Proof With the help of Lemma 1 and Lemma 2, and substituting (16) and (19) into (15), (21) is derived.

$$
\begin{aligned}
& F_{\gamma_{e}}\left(x_{0}\right)=
\end{aligned}
$$



Theorem 2 When $m_{l p}$ is an arbitrary value, $F_{\gamma_{e}}\left(x_{0}\right)$ can be given by (22).

Proof As with Theorem 1, with the help of Lemma 1 and Lemma 2, and substituting (17) and (20) into (15), (22) is derived.

$$
\begin{aligned}
& F_{\gamma_{e}}\left(x_{0}\right)=
\end{aligned}
$$






\subsection{Instantaneous capacity}

In this section, we analyze the instantaneous capacity of the system, and the analytical expression for the instantaneous capacity of the system is given. The approximate value of the system at high SNRs region is also derived.

In information-theoretical view, the instantaneous capacity has the following definition which refers to the value of the instantaneous maximum mutual information between the source and the destination, which can be mathematically formulated as

$$
C=\frac{1}{2} \log _{2}\left(1+\gamma_{e}\right)
$$

where $\gamma_{e}$ is the SINDR of the system and $C$ is the instantaneous capacity of the system.

Now, by substituting (4), (5), and (6) into (23), the instantaneous capacity can be derived as (24) which is at the top of eleventh page.

$$
\begin{gathered}
C=\frac{1}{2} \log _{2}\left(1+\gamma_{e}\right) \\
=\frac{1}{2} \log _{2}\left\{1+\min \left[\max _{i=1, \ldots, N_{1}}\left(\frac{\lambda_{1 i}}{\lambda_{1 i} \Delta_{1 i}+1}\right),\right.\right. \\
\left.\left.\frac{\lambda_{2}}{\lambda_{2} \Delta_{2}+\sum_{j=1}^{I_{1}} \lambda_{j}\left(1+\Delta_{j}\right)+1}\right]\right\}
\end{gathered}
$$

From (24), we find that, when $\lambda_{1 i}$ and $\lambda_{2}$ become infinite, (24) achieves a fixed value that is just a function of $\Delta_{1 i}$ and $\Delta_{2}$ as in (25).

$$
C=\frac{1}{2} \log _{2}\left[1+\min \left(\frac{1}{\Delta_{1 i}}, \frac{1}{\Delta_{2}}\right)\right]
$$

\section{Numerical results}

In this section, Monte Carlo simulations are provided to verify the analytical results. Some representative numerical results are provided to evaluate the impact of hardware impairments of the considered systems. Here, the analytical curves of arbitrary the $m_{l p}$ value case are obtained by using the first 30 terms of the infinite series. In this section, we assume that $m_{2}=5, b_{2}=0.251, \Omega_{2}=0.279$ for the channel parameters between $R$ and $D, k_{j}=0.1$, $P_{1 i}=P_{r}$ and $\frac{P_{j}}{\delta_{d}^{2}}=1 d B$. We also assume that $N_{1}=3$ and $I_{1}=3$ for the simulations. As shown, the analytical results agree well with the Monte Carlo simulations, which imply the correctness of the derived theoretical formula.

Figure 2 plots the outage performance of the satellite relay system with ideal hardware and impaired hardware. As shown in Fig. 2, we can see that the theory results are sufficiently tight across the entire SNR with the simulation results, which verifies the correctness of our analysis. From the results, we can also see that when the channel is under heavy fading, the system has worse performance at low SNRs. At high SNRs, the outage probability for the two conditions is the same only having a relationship with the impairment level. Moreover, from Fig. 2, we know that the lower the hardware impairments' level is, the lower the outage probabilities are.

Figure 3 examines the impact of the outage threshold on the outage performance of the considered system. It can be clearly seen that when the system has hardware impairments, the outage probability increases to 1 as the outage threshold increases to a certain constant. The larger the $k_{1 i}$ and $k_{2}$ are, the lower the outage threshold is. In contrast, when the system has ideal hardware, there exists no outage threshold for the considered system. From the results, we can also see that when the channel is under heavy fading, the system has worse performance.

Figure 4 depicts that that hardware impairments have a small impact on the instantaneous capacity at low SNRs, but are very influential at high SNRs. It also depicts that when the system has hardware impairments, the instantaneous capacity has an upper bound at high SNRs which is proved by (25). However, the instantaneous capacity at high SNRs of ideal hardware has no upper bound.

\section{Conclusions}

In this paper, we investigated dual-hop satellite relay networks with hardware impairments and co-channel interference. Specifically, the exact closed-form expression for the outage probability of the considered system was derived, which made it easy to evaluate the impact of the impairing parameters on the system performance. The results revealed that an outage floor appeared when the hardware impairments existed. In addition, the analytical expression for the instantaneous capacity was also derived, which revealed that the instantaneous capacity had an upper bound when the system had hardware impairments. However, when the channel was under heavy fading, the performance of the system was worse.

\section{Appendix}

In this appendix, the derivations of (19) and (20) are presented. First, with the help of (10) and (12), (18) can be further represented as (26) and (27), respectively. 


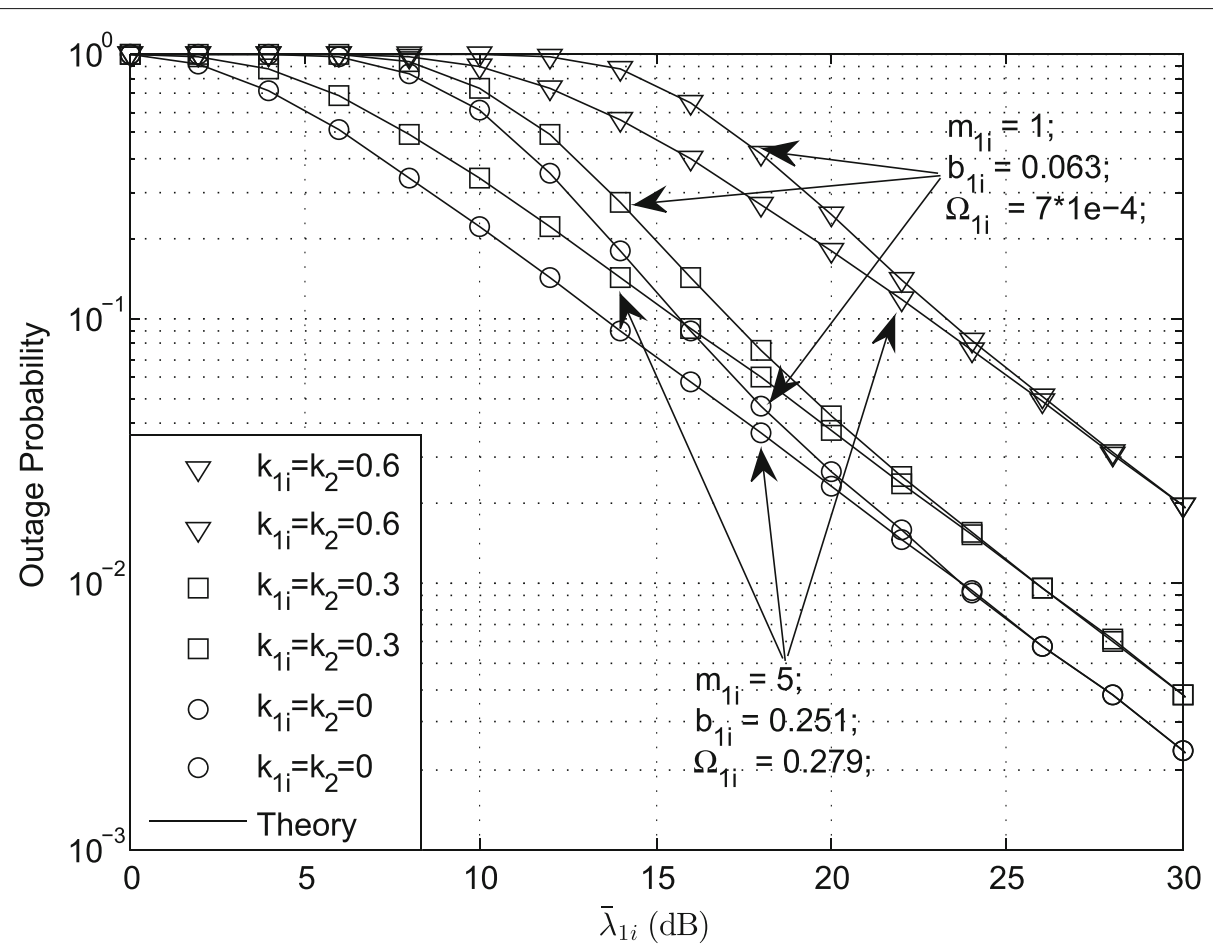

Fig. 2 Outage probability of the system with ideal hardware and hardware impairments

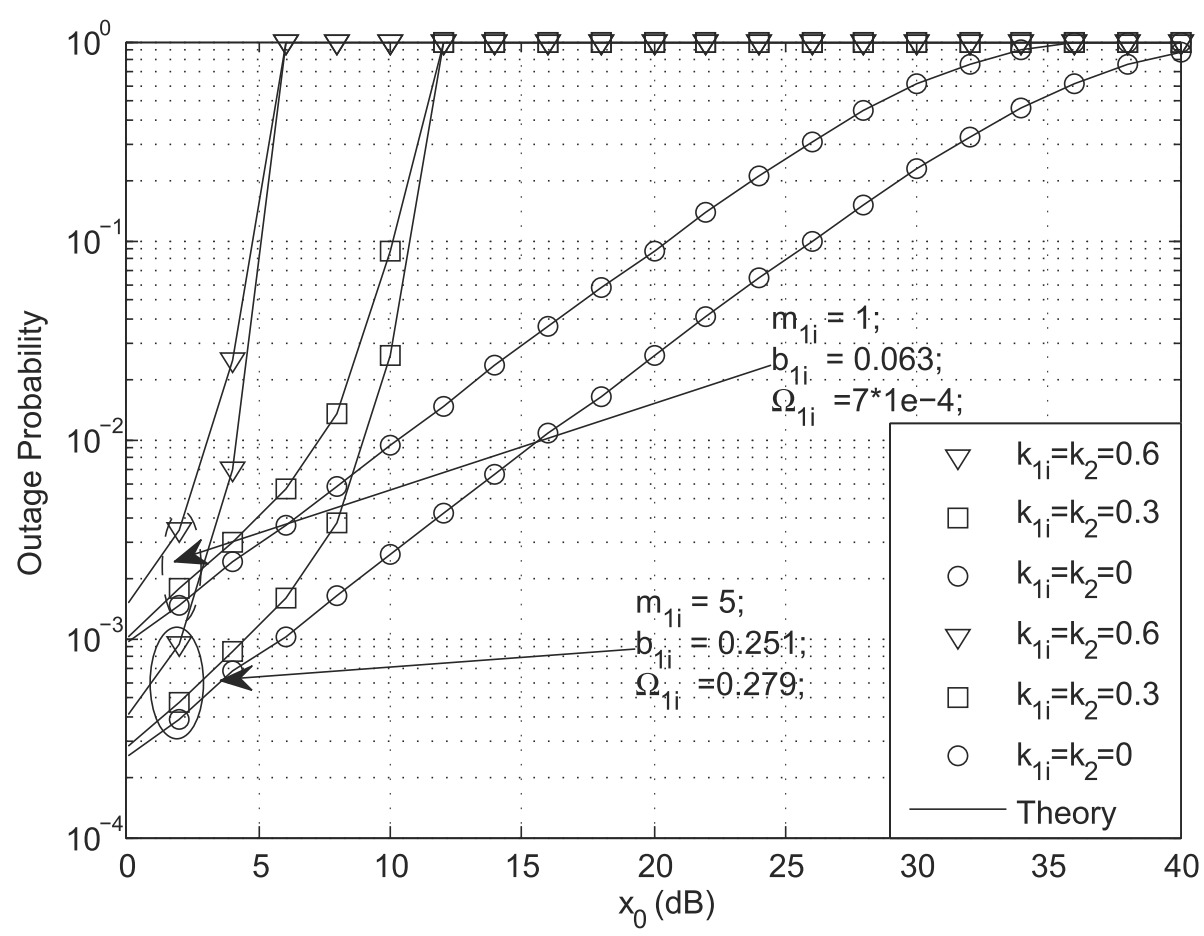

Fig. 3 Outage probability for different threshold $x_{0}$ 


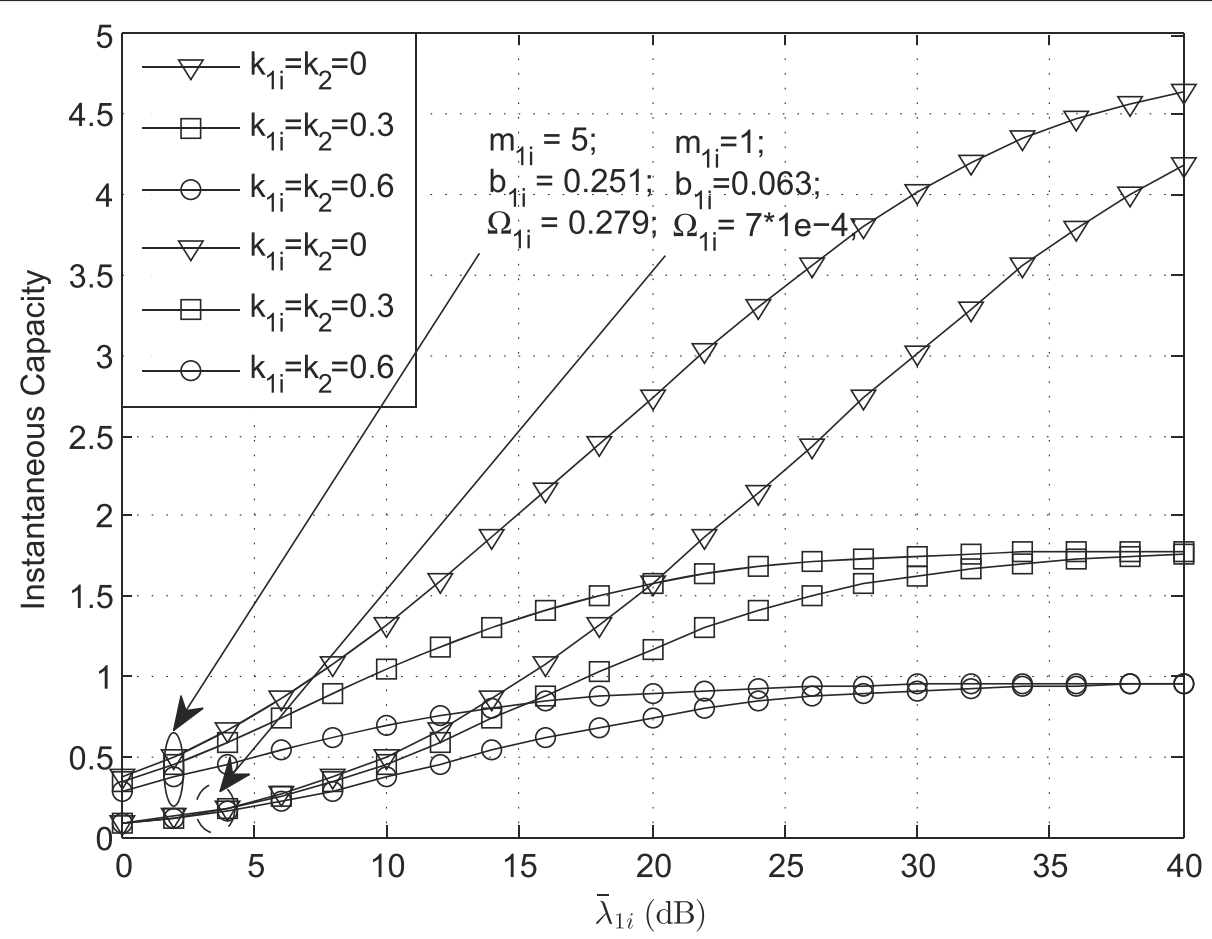

Fig. 4 The instantaneous capacity of the system

$$
\begin{aligned}
& F_{\gamma_{2}}\left(x_{0}\right)= \\
& \alpha_{2} \sum_{k_{2}=0}^{m_{2}-1} \frac{\left(-\delta_{2}\right)^{k_{2}}\left(1-m_{2}\right)_{k_{2}}}{\left(k_{2} !\right)^{2}\left(\beta_{2}-\delta_{2}\right)^{k_{2}+1}} \gamma\left(k_{2}+1,\left(\frac{\beta_{2}-\delta_{2}}{\bar{\lambda}_{2}}\right)\left[\frac{\sum_{j=1}^{I_{1}} \lambda_{j}\left(1+\Delta_{j}\right) x_{0}+x_{0}}{1-\Delta_{2} x_{0}}\right]\right) \\
& F_{\gamma_{2}}\left(x_{0}\right)=\alpha_{2} \sum_{k_{2}=0}^{\infty} \frac{\left(m_{2}\right)_{k_{2}}}{\left(k_{2} !\right)^{2}} \frac{\delta_{2}^{k_{2}}}{\beta_{2}^{k_{2}+1}} \gamma\left(k_{2}+1, \frac{\beta_{2}}{\bar{\lambda}_{2}}\left[\frac{\sum_{j=1}^{I_{1}} \lambda_{j}\left(1+\Delta_{j}\right) x_{0}+x_{0}}{1-\Delta_{2} x_{0}}\right]\right)
\end{aligned}
$$

With the help of [27. section 8.252.1] and [27. section 1.111], (26) and (27) can be expanded to (28) and (29), respectively.

$$
\begin{aligned}
& F_{\gamma_{2}}\left(x_{0}\right)= \\
& \begin{cases}\alpha_{2} \sum_{k_{2}=0}^{m_{2}-1} \frac{\left(-\delta_{2}\right)^{k_{2}}\left(1-m_{2}\right)_{k_{2}}}{\Gamma\left(k_{2}+1\right)\left(\beta_{2}-\delta_{2}\right)^{k_{2}+1}}\left\{1-\left(\frac{\beta_{2}-\delta_{2}}{\bar{\lambda}_{2}}\right)^{m} e^{-\left(\frac{\beta_{2}-\delta_{2}}{\bar{\lambda}_{2}}\right)\left(\frac{x_{0}}{1-\Delta_{2} x_{0}}\right)}\left\{1-\left[\sum_{j=1}^{I_{1}} \lambda_{j}\left(1+\Delta_{j}\right)\right]\right\}\right. \\
\left.\times\left\{\sum_{m=0}^{k_{2}} \sum_{v=0}^{m} \frac{1}{m !}\left(\begin{array}{c}
m \\
v
\end{array}\right)\left(\frac{x_{0}}{1-\Delta_{2} x_{0}}\right)^{m}\left[\sum_{j=1}^{I_{1}} \lambda_{j}\left(1+\Delta_{j}\right)\right]^{v}\right\}\right\}, & x_{0}<\frac{1}{\Delta_{2}} \\
1, & x_{0} \geq \frac{1}{\Delta_{2}}\end{cases}
\end{aligned}
$$




$$
\begin{aligned}
& F_{\gamma_{2}}\left(x_{0}\right)= \\
& \left\{\begin{array}{l}
\alpha_{2} \sum_{k_{2}=0}^{\infty} \frac{\left(m_{2}\right)_{k_{2}}}{\Gamma\left(k_{2}+1\right)} \frac{\delta_{2}^{k_{2}}}{\beta_{2}^{k_{2}+1}}\left\{1-\left(\frac{\beta_{2}}{\bar{\lambda}_{2}}\right)^{m} e^{-\left(\frac{\beta_{2}}{\bar{\lambda}_{2}}\right)\left(\frac{x_{0}}{1-\Delta_{2} x_{0}}\right)\left\{1-\left[\sum_{j=1}^{I_{1}} \lambda_{j}\left(1+\Delta_{j}\right)\right]\right\}}\right. \\
\left.\times\left\{\sum_{m=0}^{k_{2}} \sum_{v=0}^{m}\left(\begin{array}{c}
m \\
v
\end{array}\right) \frac{1}{m !}\left(\frac{x_{0}}{1-\Delta_{2} x_{0}}\right)^{m}\left[\sum_{j=1}^{I_{1}} \lambda_{j}\left(1+\Delta_{j}\right)\right]^{i i}\right\}\right\} \begin{array}{l}
, x_{0}<\frac{1}{\Delta_{2}} \\
x_{0} \geq \frac{1}{\Delta_{2}}
\end{array}
\end{array}\right.
\end{aligned}
$$

We first define $X=\sum_{j=1}^{I_{1}} \lambda_{j}$, and the PDF of $X$ can be given by [29]

$$
p_{X}(x)=\sum_{i=1}^{\rho(A)} \sum_{j=1}^{\tau_{i}(A)} \chi_{i, j}(A) \frac{\mu_{\langle i\rangle}^{-j}}{(j-1) !} x^{j-1} e^{-\frac{x}{\mu_{\langle i\rangle}}}, x \geq 0
$$

where $A=\operatorname{diag}\left(\mu_{1}, \mu_{2}, \cdots \mu_{I_{1}}\right), \rho(A)$ is the number of district diagonal elements of $A, \mu\langle 1\rangle>\mu\langle 2\rangle>\cdots>\mu\langle\rho(A)\rangle$ are the district diagonal elements in decreasing order, $\tau_{i}(A)$ is the multiplicity of $A$, and $\chi_{i, j}(A)$ is the $(i, j)$-th characteristic coefficient of $A$ [30].

From the definition of the conditional probability density function (CPDF), (18) can also be expressed as

$$
F_{\gamma_{2}}\left(x_{0}\right)=\int_{0}^{\infty} F_{\gamma_{2}}\left(x_{0} \mid x\right) p_{X}(x) d x
$$

Substituting (28), (29) and (30) into (31), and after performing some simplifications, (31) can be represented as (32) and (33).

$$
\begin{aligned}
& F_{\gamma_{2}}\left(x_{0}\right)=
\end{aligned}
$$

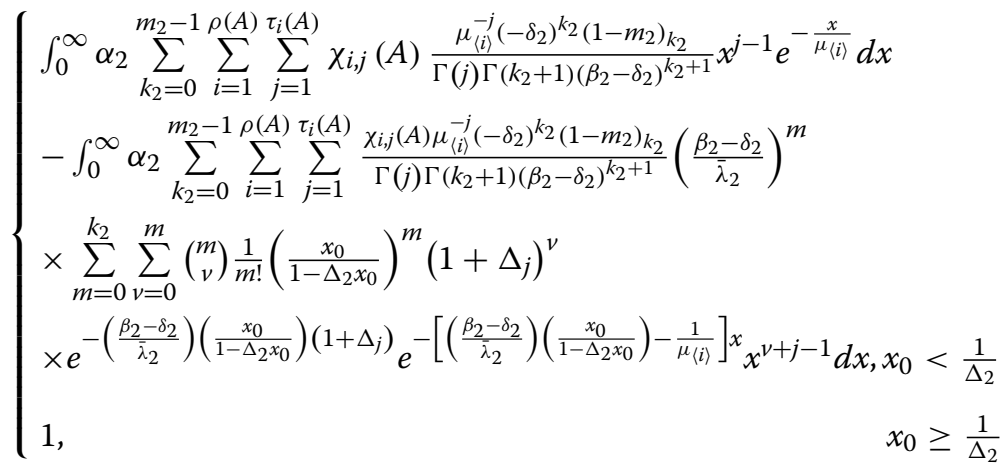

$$
\begin{aligned}
& F_{\gamma_{2}}\left(x_{0}\right)= \\
& \left\{\begin{array}{l}
\int_{0}^{\infty} \alpha_{2} \sum_{k_{2}=0}^{\infty} \sum_{i=1}^{\rho(A)} \sum_{j=1}^{\tau_{i}(A)} \frac{\chi_{i, j}(A) \mu_{\langle i\rangle}^{-j}\left(m_{2}\right)_{k_{2}} \delta_{2}^{k_{2}}}{\Gamma(j) \Gamma\left(k_{2}+1\right) \beta_{2}^{k_{2}+1}} x^{j-1} e^{-\frac{x}{\mu_{\langle i\rangle}}} d x \\
-\int_{0}^{\infty} \alpha_{2} \sum_{k_{2}=0}^{\infty} \sum_{i=1}^{\rho(A)} \sum_{j=1}^{\tau_{i}(A)} \frac{\chi_{i, j}(A) \mu_{\langle i\rangle}^{-j}\left(m_{2}\right)_{k_{2}} \delta_{2}^{k_{2}}}{\Gamma(j) \Gamma\left(k_{2}+1\right) \beta_{2}^{k_{2}+1}}\left(\frac{\beta_{2}}{\bar{\lambda}_{2}}\right)^{m} \\
\times \sum_{m=0}^{k_{2}} \sum_{\nu=0}^{m}\left(\begin{array}{c}
m \\
v
\end{array}\right) \frac{1}{m !}\left(\frac{x_{0}}{1-\Delta_{2} x_{0}}\right)^{m}\left(1+\Delta_{j}\right)^{v}
\end{array}\right. \\
& \times e^{-\left(\frac{\beta_{2}}{\lambda_{2}}\right)\left(\frac{x_{0}}{1-\Delta_{2} x_{0}}\right)\left(1+\Delta_{j}\right)} e^{-\left[\left(\frac{\beta_{2}}{\lambda_{2}}\right)\left(\frac{x_{0}}{1-\Delta_{2} x_{0}}\right)-\frac{1}{\mu_{\langle i\rangle}}\right] x} x^{\nu+j-1} d x, x_{0}<\frac{1}{\Delta_{2}} \\
& \text { 1, } \quad x_{0} \geq \frac{1}{\Delta_{2}}
\end{aligned}
$$

With the help of [27. section 3.351.3], and after simplifications, (19) and (20) are presented. 


\section{Acknowledgements}

This work is supported by the National Science Foundation of China (No.61501507) and the Jiangsu Provincial Natural Science Foundation of China (No.20150719).

\section{Competing interests}

The authors declare that they have no competing interests.

\section{Publisher's Note}

Springer Nature remains neutral with regard to jurisdictional claims in published maps and institutional affiliations.

Received: 9 May 2016 Accepted: 26 June 2017

Published online: 17 July 2017

\section{References}

1. JN Laneman, DNC Tse, GW Wornell, Cooperative diversity in wireless networks: efficient protocols and outage behavior. IEEE Trans. Inf. Theroy. 50(4), 1963-1968 (2004)

2. B Wang, Z Jun, Z Li, Achieveable rates and scaling laws of power-constrained wireless sensory relay networks. IEEE Commun.on Infor Theory. 52(9), 4084-4104 (2006)

3. KS Gomadam, SA Jafar, The effect of noise correlation in amplify-and-forward relay networks. IEEE Commun.on Infor Theory. 55(2), 731-745 (2009)

4. Y Huang, J Wang, Q Wu, C Zhong, C Li, Outage performance of spectrum sharing systems with MRC diversity under multiple primary user's interference. IEEE Commun. Lett. 16(4), 576-579 (2014)

5. A Abdi, WC Lau, MS Alouini, M Kaveh, A new simple model for land mobile satellite channels: first-and second-order statistics. IEEE Trans. Wireless Commun. 2(3), 519-528 (2003)

6. NI Miridakis, DD Vergados, A Michalas, Dual-hop communication over a satellite relay and shadowed Rician channels. IEEE Trans. Vehi. Tech. 64(9), 4031-4040 (2015)

7. S Wang, M Yang, Q Guo, in The Fourth Int. Conf. Adv. Sat. Space Commun, Chamonix. Outage probability evaluation of land mobile satellite cooperative diversity communication system (IEEE, MontBlanc, 2012), pp. 26-30

8. A lqbal, KM Ahmed, A hybrid satellite-terrestrial cooperative network over non identically distributed fading channels. J. Commun. 6(7), 581-589 (2011)

9. MR Bhatnagar, MK Arti, Performance analysis of AF based hybrid satellite-terrestrial cooperative network over generalized fading channels. IEEE Commun. Lett. 17(10), 1912-1915 (2013)

10. MR Bhatnagar, MK Arti, Performance analysis of hybrid satellite-terrestrial FSO cooperative system. IEEE Photon. Technol. Lett. 25(22), 2197-2200 (2013)

11. E Costa, S Pupolin, m-QAM-OFDM system performance in the presence of a nonlinear amplifier and phase noise. IEEE Trans.Commun. 50(3), 462-472 (2002)

12. T Schenk. RF imperfections in high-Rate wireless systems: impact and digital compensation (Springer, Netherlands, 2008), pp. 2033-2043

13. C Studer, M Wenk, A Burg, in Proc. 2010 ITG Work. Smart Ant. MIMO transmission with residual transmit-RF impairments, (Bremen, 2010), pp. 189-196

14. J Qi, S Assa, MS Alouini, in Proc. 2012 IEEE Wireless Commun. Netw. Conf Analysis and compensation of I/Q imbalance in amplify-and-forward cooperative systems ((WCNC) Pairs, France, 2012), pp. 1-4

15. E Bjornson, $\mathrm{P}$ Zetterberg, M Bengtsson, B Ottersten, Capacity limits and multiplexing gains of MIMO channels with transceiver impairments. IEEE Commun. Lett. 17(1), 91-94 (2013)

16. Z Xinlin, M Matthaiou, E Bjornson, M Coldrey, M Debbah, On the MIMO capacity with residual transceiver hardware impairments. (IEEE ICC, Jun 2014 Sydney, Australia, 2014), pp. 10-14

17. E Bjornson, J Hoydis, M Kountouris, M Debbah, in 2013 8th International Conference on Digital Signal Processing. Hardware impairments in large-scale MISO systems: energy efficiency, estimation, and capacity limits (Fira, Greece, 2013), pp. 18-20

18. XXia, D Zhang, KXu, W Ma, Y Xu, Hardware impairments aware transceiver for full-duplex massive MIMO relaying. IEEE Trans. Sign. Proc. 63(24), 6565-6580 (2015)
19. TT Duy, TQ D, DB Costa, VNQ Bao, M Elkashlan, Proactive relay selection with joint impact of hardware impairment and co-channel interference. IEEE Trans. Commun. 63(5), 1594-1606 (2015)

20. M Maletic, M Cabarkapa, N Neskovic, D Budimir, Hardware impairments impact on fixed-gain AF relaying performance in Nakagami-m fading. Electronics Lett. 52(2), 121-122 (2016)

21. K An, M Lin, J Ouyang, H Wei, Performance analysis of beamforming in two-hop AF relay networks with antenna correlation and interference. AEU-Int. J. Electron. Commun. 68(7), 587-594 (2014)

22. C Zhong, S Jin, KK Wong, Dual-hop systems with noisy relay and interference-limited destination. IEEE Trans. Commun. 58(3), 764-768 (2010)

23. YZ Huang, FA Qahtani, C Zhong, Q Wu, J Wang, H Alnuweiri, Performance analysis of multiuser multiple antenna relaying networks with co-channel interference and feedback delay. IEEE Trans. Commun. 62(1), 59-73 (2014)

24. M Sadek, S Aissa, Personal satellite communication: technologies and challenges. IEEE Wireless Commun. 12(5), 28-35 (2012)

25. K An, M Lin, J Ouyang, Y Huang, G Zheng, Symbol error analysis of hybrid satellite-terrestrial cooperative networks with cochannel interference. IEEE Commun. Lett. 18(11), 1947-1950 (2014)

26. 8 hints for making and interpreting EVM measurements (2005). Tech. Rep. Agilent Technologies

27. IS Gradshteyn, IM Ryzhik, Table of Intergals Series and Products, 7th ed. (Elsevier, Amsterdam, 2005), pp. 5989-3144EN

28. The Wolfram Function Site. [Online], Avilable: http://functions.wolfram com. Accessed 1998

29. A Bletsas, H Shin, MZ Win, Cooperative communication with outage-optimal opportunistic relaying. IEEE Trans. Wire. Commun. 6(9), 3450-3460 (2007)

30. H Shin, MZ Win, MIMO diversity in the presence of double scattering. IEEE Trans. Inform. Theory. 54(7), 2976-2996 (2008)

\section{Submit your manuscript to a SpringerOpen ${ }^{\circ}$ journal and benefit from:}

Convenient online submission

- Rigorous peer review

- Open access: articles freely available online

- High visibility within the field

Retaining the copyright to your article

Submit your next manuscript at $\$$ springeropen.com 